\title{
Non trivial influence of acoustic streaming on the efficiency of annular thermoacoustic prime
}

\section{movers}

\author{
G. Penelet ${ }^{\mathrm{a}, *}$ V. Gusev ${ }^{\mathrm{a}, \mathrm{b}}$ P. Lotton ${ }^{\mathrm{a}}$ M. Bruneau ${ }^{\mathrm{a}}$ \\ ${ }^{a}$ Laboratoire d'Acoustique de l'Université du Maine, UMR CNRS 6613 \\ Avenue Olivier Messiaen, 72085 Le Mans Cedex 9, France \\ ${ }^{\mathrm{b}}$ Laboratoire de Physique de l'Etat Condensé, UMR CNRS 6087 \\ Avenue Olivier Messiaen, 72085 Le Mans Cedex 9, France
}

\begin{abstract}
The nonlinear processes controlling the time-dependent evolution of sound in annular thermoacoustic prime movers are studied. It is demonstrated that, under some heating conditions, the evolution of the temperature field induced by the excitation of acoustic streaming provides an additional amplification of sound which results in a complicated periodic onset and damping of thermoacoustic instability. The study of this particular example provides the opportunity to demonstrate that the excitation of acoustic streaming does not necessarily imply a decrease in the engine's efficiency.
\end{abstract}

Key words: Thermoacoustics, nonlinear acoustics, acoustic streaming PACS: 43.35.Ud, 43.25.Nm

\footnotetext{
* corresponding author.

Email address: guillaume.penelet@univ-lemans.fr (G. Penelet).
} 


\section{Introduction}

Thermal interactions between solid walls and oscillating gas are responsible for various thermoacoustic phenomena such as self sustained acoustic waves in resonators or acoustic heat pumping. Since the early 80 's, there has been a renewal of interest in thermoacoustic devices, both prime movers (i.e. thermal to acoustic energy converters) and heat pumps, so that a great variety of devices has been developed $[1,2]$. The linear analytical theory of thermoacoustics is now well developed $[1,3]$, but not sufficient to understand the great complexity of such heat engines. In particular, it is now well established that steady-state sound in thermoacoustic prime movers is controlled by a few nonlinear effects, but it is however difficult both to predict the acoustic pressure amplitude in the steady regime and to clearly understand the processes controlling the dynamics of acoustic pressure amplitude evolution observed experimentally, even in very simple thermoacoustic devices. Among the mechanisms saturating the wave amplitude growth is the excitation of acoustic streaming [4,5], i.e. the acoustically induced generation of a mean (non oscillating) mass flow, which influences the temperature distribution in the device. Though an important research effort has recently been devoted to the description of acoustic streaming in resonant tubes [6-11] and thermoacoustic devices [12-14], the role of this mechanism in thermoacoustic prime movers is poorly understood : its presence has been evidenced in various thermoacoustic devices [15-19], but the physics of its influence on the saturation of the wave amplitude growth in the prime movers have not yet been completely explained. However, it seems to be commonly acknowledged that forced convection due to acoustic streaming has a harmful influence on the efficiency of thermoacoustic engines because it tends 
to reduce the externally imposed temperature gradient along the stack, and because of this, various experimental solutions have been proposed to eliminate this acoustic streaming $[16,17]$. The aim of this letter is to question (or at least to moderate) this conclusion by the experimental and theoretical study of a particular example, and to demonstrate that, under some circumstances, acoustic streaming can improve the efficiency of thermoacoustic prime movers.

\section{Experiments}

The thermoacoustic device which is studied here is presented schematically in Fig. 1 (a): this is an annular thermoacoustic prime mover. It is basically composed of a resonant annular tube and a stack of solid plates submitted to a strong temperature gradient. The torus-shaped stainless steel tube of length $L=2.24 \mathrm{~m}$ is filled with air at atmospheric pressure $p_{0} \approx 10^{5} \mathrm{~Pa}$. The stack (length $H_{s}=5 \mathrm{~cm}$ ) is a honeycombed ceramic material with square channels of cross-section $0.9 \times 0.9 \mathrm{~mm}^{2}$. The cold heat exchanger consists of a copper circular shell set around the tube with flowing cooling water inside at room temperature $T_{\infty}$, and a copper wire mesh in the tube (attached to the left side of the stack). In order to impose a strong temperature gradient along the stack, a 1 centimeter thick hot heat exchanger is placed in the waveguide $5 \mathrm{~mm}$ away from the right side of stack. This hot heat exchanger is made of the same honeycombed ceramic material, used here as a skeleton for the coiling of a nichrome wire (i.e. an electrical heat resistance) connected to a thyristor unit. In such a device, when the temperature gradient along the stack exceeds a certain critical value, the thermoacoustic amplification process (which occurs in the stack, inside thermal boundary layers) results in the self 
excitation of high amplitude resonant acoustic waves. This is called the onset of thermoacoustic instability. For each of the measurements presented in the following, the heating power supply is preliminarily set to $Q_{0}=54 \mathrm{~W}$ so that the device is just below the onset of thermoacoustic instability. A small $\Delta Q$ increment is then sufficient for the acoustic wave to be generated in the waveguide at frequency $f \approx 153 \mathrm{~Hz}$, which corresponds approximately to the frequency of the first travelling wave mode of the annular resonator $(f \approx$ $c / L$, where $c \approx 340 \mathrm{~m} \cdot \mathrm{s}^{-1}$ denotes the adiabatic sound velocity). Note that care was taken before each measurement to wait for temperature stabilization throughout the device.

It is schematically shown in Fig. 1(b) that the temperature $T_{H}$ at the hot stack end is measured using a type $\mathrm{K}$ thermocouple probe $(70 \mu \mathrm{m}$ in diameter) glued with a ceramic putty onto the stack. The amplitude of acoustic pressure oscillations $p_{1}$ is measured using a piezoresistive microphone placed approximately 1 metre away from the hot stack end. Note that subscript "1" is used in this paper as a reminder that acoustic pressure is a quantity of the first order of smallness in comparison with static pressure $\left(p_{1}<<p_{0}\right)$.

In Fig. 2, the evolutions with time of both acoustic pressure amplitude $p_{1}$ and temperature at the hot stack end $T_{H}$ are presented (respectively in solid and dashed lines), for different values of the heating power increment $\Delta Q$. Regarding the evolution of $p_{1}$, the results show the possibility for the device to turn on and off spontaneously and periodically, and the switch on-off period decreases when the $\Delta Q$ increment increases (Fig. 2 (a),(b),(c)). Then, when $\Delta Q / Q_{0}$ exceeds $30 \%$, the amplitude of the acoustic waves finally stabilizes to a finite value (Fig. $2(\mathrm{~d})$ ). Regarding the evolution of the hot stack end temperature, it is remarkable that the variations of $T_{H}$ are strongly coupled 
to those of $p_{1}$ : for small $\Delta Q$ increments, periodic evolution of $T_{H}$ is observed, while for larger $\Delta Q$ the stabilization of the acoustic pressure amplitude is accompanied by the stabilization of $T_{H}$. These results indicate that the nonlinear effects involving the reverse influence of the acoustic field on the temperature field may play a predominant role in the observed periodic onset and damping of thermoacoustic instability: this behavior seems to be the consequence of a competition between heating (with subsequent rise of $T_{H}$ ) inducing thermoacoustic amplification of sound and acoustically induced heat transport tending to reduce the temperature difference between stack ends (with subsequent attenuation of sound). However, it is remarkable in Fig. 2 (d) that the temperature $T_{H}$ before the first onset of thermoacoustic instability (i.e. at time $t \approx 0$ in Fig. $2(\mathrm{~d}))$ is more than 30 degrees higher than its value after stabilization of the acoustic pressure amplitude to a finite - but nonzero - value. The observation that oscillations might be sustained by smaller temperature gradients than those necessary to initiate instability indicates that not only the temperature difference between stack ends but also the shape of the temperature field throughout the heterogeneously heated part of the device impact the thermoacoustic amplification process.

None of the observations described above can be considered as fundamentally new in the field of thermoacoustics. Such regimes of periodic onset and damping of thermoacoustic instability have already been mentioned $[22,23]$ in standing wave thermoacoustic devices, and other phenomena involving the concept of multistability have been reported [24,25]. Moreover, the influence of the shape of the temperature distribution on the thermoacoustic amplification of sound in annular thermoacoustic prime movers has been recently demonstrated [20]. However, to our knowledge, the physical explanation of such an 
onset/damping behavior has not been clearly provided. The theoretical analysis presented below aims at filling this gap.

\section{$3 \quad$ Analytical model}

The time-dependent regime of the prime mover operation is modelled here by combining the equation describing the variations of acoustic pressure amplitude with the equations describing, in a simplified one dimensional approach, the heat transfer through the heterogeneously heated parts of the device (which will be called "thermoacoustic core" in the remaining of the

paper). Only the general outlines of the model are presented here, because the complete analytical approach can be found in previous papers [20,21], devoted to the analysis of processes different from those analyzed here (due to differences in heating geometry).

Heat transfer throughout the thermoacoustic core is described by the coupling between three homogeneous media, which are, as depicted in Fig. 3, the stainless steel resonator walls (with the corresponding temperature field $T_{r}(x, t)$ ), the air in the heterogeneously heated part of the waveguide $\left(T_{\mathrm{w}}(x, t)\right)$, and the stack (composed both of air and ceramics, but considered as a homogeneous medium at temperature $T_{s}(x, t)$ in the heat transfer equations). This results in the following set of equations:

$$
\begin{gathered}
x \in\left[-H_{S}, 0\right], \partial_{t} T_{r}=\kappa_{r} \partial_{x x}^{2} T_{r}-\gamma^{r \rightarrow s}\left(T_{r}-T_{s}\right)-\gamma^{r \rightarrow \infty}\left(T_{r}-T_{\infty}\right), \\
\partial_{t} T_{s}+v_{s} \partial_{x} T_{s}=\kappa_{s} \partial_{x x}^{2} T_{s}-\gamma^{s \rightarrow r}\left(T_{s}-T_{r}\right), \\
x \in\left[0, H_{W}\right], \partial_{t} T_{r}=\kappa_{r} \partial_{x x}^{2} T_{r}-\gamma^{r \rightarrow \mathrm{w}}\left(T_{r}-T_{\mathrm{w}}\right)-\gamma^{r \rightarrow \infty}\left(T_{r}-T_{\infty}\right), \\
\partial_{t} T_{\mathrm{w}}+v_{\mathrm{w}} \partial_{x} T_{\mathrm{w}}=\kappa_{\mathrm{w}} \partial_{x x}^{2} T_{\mathrm{w}}-\gamma^{\mathrm{w} \rightarrow r}\left(T_{\mathrm{w}}-T_{r}\right),
\end{gathered}
$$


where $\partial_{t}$ and $\partial_{x}$ stand for partial time and space derivative respectively, and where $\kappa_{r, s, \mathrm{w}}$ denote thermal diffusivities of the media. Several points need to be explained concerning Eqs. (1).

First of all, the coupling coefficients $\gamma^{r \leftrightarrow s}, \gamma^{r \leftrightarrow \mathrm{w}}$ and $\gamma^{r \rightarrow \infty}$ are approximate phenomenological coefficients obtained from heat transfer theory [29]. The calculation details leading to the analytical expressions for $\gamma^{r \leftrightarrow \mathrm{w}, r \leftrightarrow s, r \rightarrow \infty}$ coefficients can be found in ref. [21].

Secondly, the advection terms $v_{s, \mathrm{w}} \partial_{x} T_{s, \mathrm{w}}$ in Eqs. $(1 \mathrm{~b}, 1 \mathrm{~d})$ account for the effect of acoustic streaming, the mean flow velocities $v_{s, \mathrm{w}}$ being controlled by the acoustic pressure amplitude. It should be mentioned that equating the effect of acoustic streaming with a unidirectional mass flow is an approximation, because the actual velocity distribution is much more complex (in particular there are upward and downward streaming currents across the duct $[19,8]$ ). However, it is well established that in annular thermoacoustic prime movers (and in other thermoacoustic devices where a closed loop path exists) there is a mean nonzero mass flow through the duct (directed clockwise in Fig. 1(a)), sometimes called "Gedeon streaming" [26], which is supposed here to provide the most important share of convective heat transfer. From previous theoretical $[12,8]$ and experimental $[18]$ works, it is then possible to derive a simplified analytical expression for the velocity $v_{s, \mathrm{w}}(t)$ of acoustic streaming [21]:

$$
d_{t} v_{s, \mathrm{w}}+\frac{v_{s, \mathrm{w}}}{\tau_{v}}=\frac{\Gamma_{v}^{s, w}}{\tau_{v}} p_{1}^{2}
$$

where it is proportional to the square of acoustic pressure amplitude, taking into account a delay $\tau_{v}$ in its onset. 
Thirdly, another effect involving the reverse influence of the acoustic field on the temperature field is hidden in Eq. (1b). This is the well-known thermoacoustic enthalpy flow induced by gas oscillations [3,27], which in the present case tends to reduce the temperature gradient. This nonlinear effect is equivalent to an acoustically enhanced thermal conductivity $[27,28]$ of the gas in the vicinity of the solid surfaces, so that in Eq. (1b), the thermal diffusivity $\kappa_{s}$ is actually separated into a static component $\kappa_{s_{0}}$ (i.e. without acoustic oscillations) and a dynamic one as follows:

$$
\kappa_{s}=\kappa_{s_{0}}+\Gamma_{\kappa} p_{1}^{2}
$$

In addition to Eqs. 1 describing heat balance in the thermoacoustic core, the following boundary conditions are considered

$$
\begin{gathered}
T_{r, s}\left(-H_{S}, t\right)=T_{\infty} \\
T_{r, \mathrm{w}}\left(H_{W}, t\right)=T_{\infty}
\end{gathered}
$$

which assume constant temperature $T_{\infty}$ at the ends of the thermoacoustic core, and

$$
\begin{aligned}
\partial_{x} T_{r}\left(0^{-}, t\right) & =\partial_{x} T_{r}\left(0^{+}, t\right), \\
\lambda_{s} \partial_{x} T_{s}\left(0^{-}, t\right) & =\lambda_{\mathrm{w}} \partial_{x} T_{\mathrm{w}}\left(0^{+}, t\right)+\frac{Q(t)}{\pi r_{i}^{2}}
\end{aligned}
$$

describing continuity of heat flux in the resonator walls and at the stack/air interface, where $\lambda_{s, \mathrm{w}}$ denotes thermal conductivity of the medium. Note that the electrical heating of the stack is modelled here simply by a homogeneous source of heat flux located at the stack/air interface, and that the thermal conductivity of the stack $\lambda_{s}$ consists of a static component and an acoustically enhanced one $\left(\lambda_{s}=\lambda_{s_{0}}+\Gamma_{\lambda} p_{1}^{2}\right.$, accordingly with Eq. (3)). 
In addition to the effects involving the reverse influence of the acoustic field on the temperature field, the saturation of the acoustic wave may also occur as a consequence of more usual nonlinear effects such as higher harmonics generation and minor losses. Both processes can be included as dissipation processes in the following set of equations describing the evolution of acoustic pressure amplitude [21]:

$$
\begin{aligned}
& d_{t} p_{1}=\Omega_{a m p l} p_{1}-\Omega_{c a s c} p_{1} \cdot p_{2}-\Omega_{\text {minor }} p_{1}^{2}, \\
& d_{t} p_{2}=\Omega_{\text {casc }} p_{1}^{2}-\Omega_{\text {diss }} p_{2},
\end{aligned}
$$

where $p_{2}$ corresponds to the second harmonic component of acoustic pressure oscillations $\left(p_{2}<<p_{1}\right)$ generated by usual nonlinear effects [5]. The first equation describes thermoacoustic amplification of the fundamental component of acoustic pressure $\left(\Omega_{a m p l} p_{1}\right)$, energy transfer from the fundamental wave to second harmonic $\left(-\Omega_{\text {casc }} p_{1} \cdot p_{2}\right)$, and dissipation of energy due to vortex generation at the edges of the stack $\left(-\Omega_{\text {minor }} p_{1}^{2}\right)$. The second equation (Eq. (5b)) describes both the generation of second harmonic $\left(\Omega_{c a s c} p_{1}^{2}\right)$ and its dissipation in the acoustic boundary layers $\left(\Omega_{d i s s} p_{2}\right)$. Importantly, it should be mentioned that the value of the amplification parameter $\Omega_{a m p l}$ depends on the temperature distribution $T_{s, \mathrm{w}}$ in the entire thermoacoustic core [20]. In particular, it has been demonstrated that, for a fixed temperature difference between stack ends, variations in the temperature distribution $T_{\mathrm{w}}(x)$ in the "passive" part of the thermoacoustic core can provide significant additional thermoacoustic amplification [20, Fig.15] by inducing (via mixing of counterpropagating acoustic waves) variations in the spatial distribution of acoustic pressure amplitude, acoustic velocity amplitude, and phase shift between pressure and velocity oscillations in the stack (i.e. in the "active" part of the thermoacoustic core where sound is amplified) [20, Fig.14], with subsequent variations in the heat 
engine's cycle.

So, while Eqs. (1) are coupled to Eqs. (5) through the effects of acoustic streaming and acoustically enhanced thermal diffusivity, Eqs. (5) are coupled to Eqs. (1) through parameter $\Omega_{a m p l}\left\{T_{s, \mathrm{w}}\right\}$. Then, the spontaneous generation and saturation of sound in the annular thermoacoustic prime mover can be computed, using classical finite difference methods [30] to solve the above listed set of nonlinear differential equations. Further details concerning the solving process, the values for geometrical and thermophysical properties of materials, and the estimates for the nonlinear parameters $\left(\Gamma_{v, \kappa}, \Omega_{c a s c, m i n o r, d i s s}, \tau_{v}\right)$ can be found in ref. [21], while the method used to calculate $\Omega_{a m p l}\left\{T_{s, \mathrm{w}}\right\}$ is presented in ref. [20].

\section{Results, discussion}

In the simulation results presented below, the protocol is analogous to the one used in experiments : the heating power is initially set to a fixed value $Q_{0}$ in order for the system to be just below threshold after computing the associated steady thermal field, and various $\Delta Q$ increments are then applied so that the onset occurs. Figure 4 presents the results obtained when $\Delta Q / Q_{0}$ increases from $5 \%$ to $30 \%$. Both the gradual evolutions of acoustic pressure amplitude $p_{1}$ and temperature at hot stack end $T_{H}$ are presented.

Comparing the results of Fig. 4 to those of Fig. 2, one might conclude that the model does not reproduce the experimental results very well : both the evolutions of $p_{1}$ and $T_{H}$ are much more abrupt in the simulations than in the experiments. Several of the assumptions made in the model could cause such a 
difference, but the most critical one is probably the one dimensional approach in the description of heat transfer. Such an approach is indeed valid if the radial temperature distribution is close to uniform : this is not the case in our experiments, where for instance a temperature difference of more than $100 \mathrm{~K}$ has been measured (just below threshold) between the temperature $T_{H}$ and the temperature at same position $x=0$ but $20 \mathrm{~mm}$ away from the stack's median axis. Note that in the paper where the analytical model of section 3 is extensively presented [21], both quantitative and qualitative agreement has been found between simulation results and experimental observations when the temperature gradient along the stack is imposed by heating the stainless steel resonator walls ; in that case, where the obtained dynamics of acoustic pressure evolution strongly differ from those observed in the present case (with "internal" heating), the temperature field inside the resonator is mostly controlled by the resonator walls (because of their high thermal inertia) and the radial temperature distribution can be considered as uniform.

Once it is clear that the results depicted in Fig. 4 must be considered only as qualitative ones, it is remarkable to see that the periodic onset/damping of thermoacoustic instability is reproduced by the model. Moreover, the stabilization of acoustic pressure amplitude to a finite value is also reproduced by the model when $\Delta Q / Q_{0}$ is sufficiently high. The results obtained thus provide the opportunity to give a physical explanation of the observed dynamics, and deeper understanding can be reached from the analysis of the time-dependent temperature field.

Figure 5 presents different temperature distributions corresponding to different stages of the wave amplitude evolution, calculated for $\Delta Q / Q_{0}=10 \%$. From the comparison of the temperature field $T_{s, \mathrm{w}}(x, t=25 \mathrm{~s})$, corresponding 
to the beginning of the onset, to the temperature field $T_{s, \mathrm{w}}(x, t=43 \mathrm{~s})$, it appears that while acoustic pressure strongly increases at time $t=43 \mathrm{~s}$, the hot temperature $T_{H}$ has already dramatically decreased. The only way to explain such results is that the excitation of acoustic streaming has changed the shape of the temperature field in such a way that additional thermoacoustic amplification has occurred. This may well be the most important thing to understand from the analysis of Fig. 5. Then, the only interest in the analysis of the other temperature fields depicted in Fig. 5 is to illustrate that the observed dynamics of acoustic pressure evolution result from a competition between heating (tending to increase $T_{H}$, with subsequent increase of $p_{1}$ ) and acoustically induced heat transfer (tending to reduce $T_{H}$, with subsequent decrease of $p_{1}$ ) including streaming induced changes of the temperature field (which influence thermoacoustic amplification in a non trivial way [20]). Importantly, while the gradual evolution of $p_{1}$ is instantaneously controlled by the temperature field $T_{s, \mathrm{w}}$ (through the parameter $\Omega_{a m p l}\left\{T_{s, \mathrm{w}}\right\}$ in Eq. (5a)), the changes of $T_{s, \mathrm{w}}$ due to heating or nonlinear effects occur with different time delays, and that may be the reason why such complicated dynamic behavior is observed.

A question naturally arises from the previous analysis : does the excitation of acoustic streaming necessarily lower the engine's efficiency? In the present case, the answer is no. Indeed, Fig. 6 presents the simulation results when the effect of acoustic streaming is ignored in the model (solid line) compared to the case when it is included in the model (dashed line) : the acoustic pressure amplitude in the steady regime is twice as high with than without taking into account acoustic streaming. More generally, the answer is probably that the effect of acoustic streaming depends on the thermoacoustic device itself, but an important, very practical conclusion of the results presented in this letter 
is that acoustic streaming may, in theory, play a favorable role if it contributes to tuning the shape of the temperature field throughout the heterogeneously heated part of the device in such a way that thermoacoustic amplification in the stack is optimized. It should be mentioned that this idea may already have been explored implicitly by Backhaus et al. $[17,31]$ in their development of a thermoacoustic Stirling engine able to reach $41 \%$ of the Carnot efficiency. The orientation of the engine relative to the gravity field, the use of a "secondary" cold heat exchanger and of a "thermal buffer tube", the use of a "jet pump" to diminish the effect of acoustic streaming, all of these experimental solutions, in addition to their effects cited in $[17,31]$, may also contribute to keeping the shape of the temperature field close to "optimum".

\section{Conclusion}

In this letter, the experimental observation in an annular thermoacoustic prime mover of a complicated periodic onset/damping of thermoacoustic instability is reported. Based on a simplified analytical model exhaustively presented in a previous paper [21], simulations are then performed, and the results reproduce the experiments qualitatively. The complicated dynamics of prime mover operation are attributed to a competition between the stack heating (tending to increase the temperature difference along the stack) and nonlinear processes involving heat transfer through the thermoacoustic core (and in particular acoustic streaming), each of these mechanisms operating with different time scales. It is also demonstrated that, in the present case, acoustic streaming tends to tune the shape of the temperature field in such a way that additional thermoacoustic amplification occurs, so that the resulting acoustic 
pressure amplitude is higher with than without streaming. In general, it is not clear how much of an effect acoustic streaming can have on the efficiency of thermoacoustic prime movers, but it seems that eliminating the mass flow associated with acoustic streaming is not necessarily the best solution. In fact, making use of acoustic streaming and/or natural convection in order to tune the temperature field "optimally" may help to reach better efficiency.

\section{References}

[1] G. W. Swift, J. Acoust. Soc. Am., 84 (1988) 1145.

[2] S. L. Garrett, Am. Journ. Phys., 72 (2004) 11.

[3] N. Rott, Adv. Appl. Mech., 20 (1980) 135.

[4] W. L. Nyborg, Physical Acoustics, Academic, New York, 1965.

[5] O. V. Rudenko, S. I. Soluyan, Theoretical Fundations on Nonlinear Acoustics, Consultants Bureau, New York, 1978.

[6] L. Menguy, J. Gilbert, Acust. Acta Acust. 86 (2000) 249.

[7] M. F. Hamilton, Y. A. Ilinskii, E. A. Zabolotskaya, J. Acoust. Soc. Am., 113 (2003) 153.

[8] M. Amari, V. Gusev, N. Joly, Acust. Acta Acust. 89 (2003) 1008.

[9] M. K. Aktas, B. Farouk, J. Acoust. Soc. Am., 116 (2004) 2822.

[10] M. W. Thompson, A. A. Atchley, J. Acoust. Soc. Am., 117 (2005) 1828.

[11] M. W. Thompson, A. A. Atchley, M. J. Maccarone, J. Acoust. Soc. Am., 117 (2005) 1839. 
[12] V. Gusev, S. Job, H. Bailliet, P. Lotton, M. Bruneau, J. Acoust. Soc. Am., 108 (2000) 934 .

[13] H. Bailliet, V. Gusev, R. Raspet, R. A. Hiller, J. Acoust. Soc. Am., 110 (2001) 2678.

[14] M. Mironov, V. Gusev, Y. Auregan, P. Lotton, M. Bruneau, P. Piatakov, J. Acoust. Soc. Am., 112 (2002) 441.

[15] T. Yazaki, A. Tominaga, Proc. Roy. Soc. Lond., ser. A 454 (1998) 2113.

[16] G. W. Swift, D.L Gardner., S. Backhaus, J. Acoust. Soc. Am., 105 (1999) 711.

[17] S. Backhaus, G.W. Swift, "A thermoacoustic Stirling heat engine", Nature, 399 (1999) 335 .

[18] S. Job, V. Gusev, P. Lotton, M. Bruneau, J. Acoust. Soc. Am., 113 (2003) 1892.

[19] Y. Ueda, T. Biwa, U Mizutani., J. Acoust. Soc. Am., 115 (2004) 1134.

[20] G. Penelet, S. Job, V. Gusev, P. Lotton, M. Bruneau, Acust. Acta Acust., 91 (2005) 567.

[21] G. Penelet, V. Gusev, P. Lotton, M. Bruneau, Phys. Rev. E, 72 (2005) 016625.

[22] G. W. Swift, J. Acoust. Soc. Am., 92 (1992) 1551.

[23] S. L. Zhou, Y. Matsubara, Cryogenics, 38 (1998) 813.

[24] G. B. Chen, T. Jin, Cryogenics, 39 (1999) 843.

[25] G. Penelet, E. Gaviot, V. Gusev, P. Lotton, M. Bruneau, Cryogenics, 42 (2002) 527.

[26] D. Gedeon, Cryocoolers, 9 (1997) 385.

[27] T. Yazaki, A. Tominaga, Y. Narahara, Journ. Heat. Transf. 105 (1983) 889.

[28] V. Gusev, H. Bailliet, P. Lotton, M. Bruneau, Acust. Acta Acust. 86 (2000) 25. 
[29] W. H. Mac Adams, Heat Transmission, $3^{\text {rd }}$ ed., 1953.

[30] M. N. Ozisik, Finite difference methods in heat transfer, CRC, Boca Raton, 1994.

[31] S. Backhaus, G.W. Swift, J. Acoust. Soc. Am., 107 (2000) 3148. 


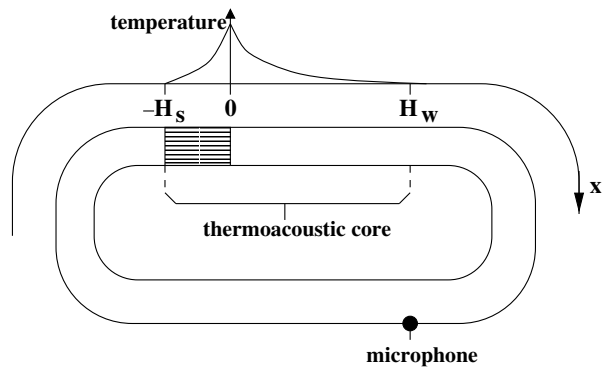

(a)

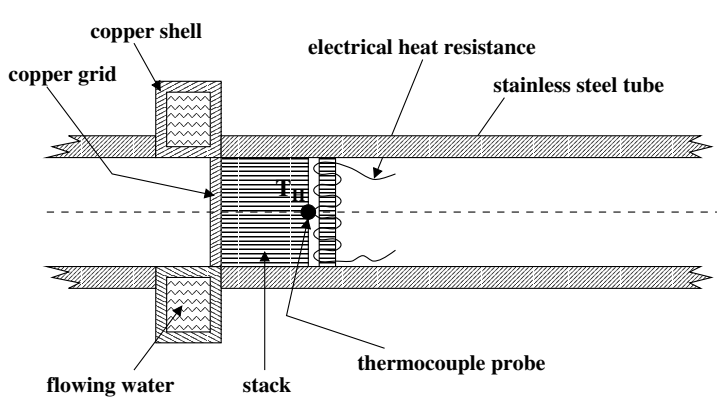

(b)

Fig. 1. (a) Schematic diagram of the experimental apparatus (b) Detailed representation of the thermoacoustic core 


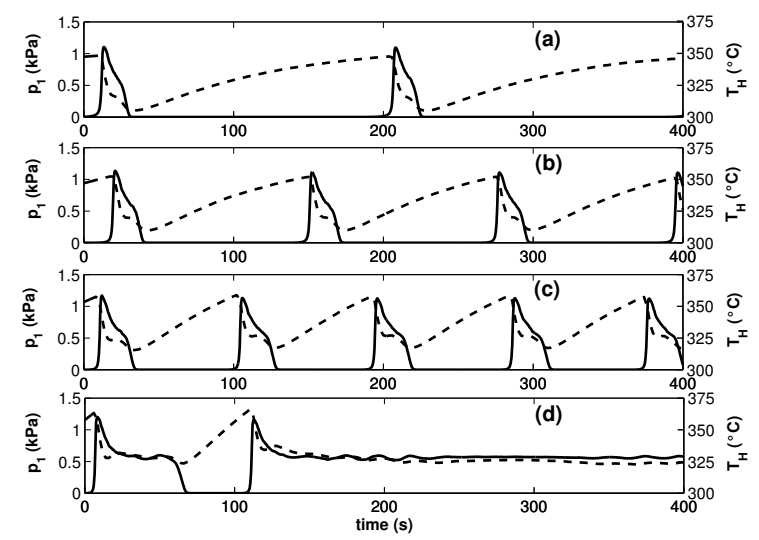

Fig. 2. Gradual evolution of the root mean square amplitude $p_{1}$ of acoustic pressure (solid line) and temperature at the hot stack end $T_{H}$ (dashed line), when the power increment $\Delta Q$ above the threshold value $Q_{0}$ is increased. (a) $\Delta Q / Q_{0}=5 \%$; (b) $\Delta Q / Q_{0}=10 \% ;$ (c) $\Delta Q / Q_{0}=20 \% ;$ (d) $\Delta Q / Q_{0}=30 \%$. 


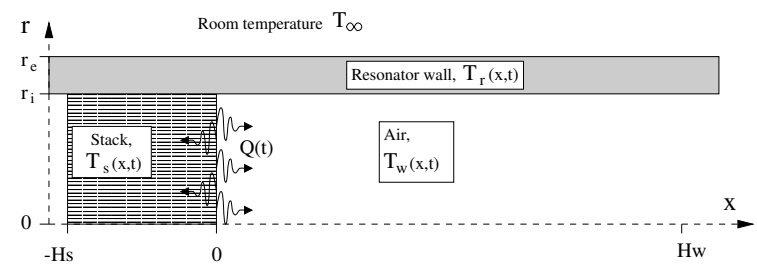

Fig. 3. Schematic representation of the thermoacoustic core, composed of three distinct homogeneous media 


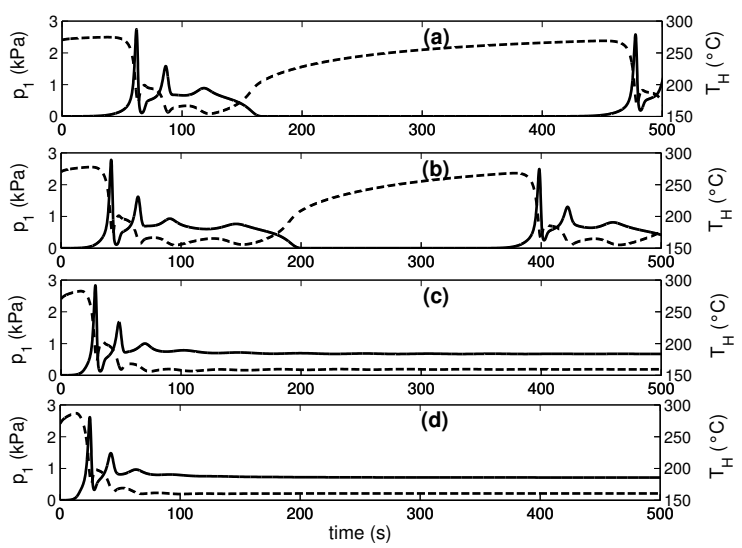

Fig. 4. Calculated evolution of the amplitude $p_{1}$ of acoustic pressure (solid line) and temperature at the hot stack end $T_{H}$ (dashed line), when the power increment $\Delta Q$ above the threshold value $Q_{0}$ is increased. (a) $\Delta Q / Q_{0}=5 \%$; (b) $\Delta Q / Q_{0}=10 \%$; (c) $\Delta Q / Q_{0}=20 \% ;$ (d) $\Delta Q / Q_{0}=30 \%$. 

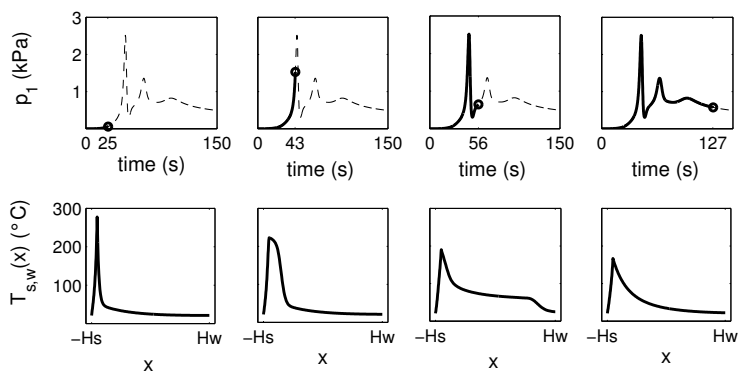

Fig. 5. Distributions of the temperature field $T_{s, \mathrm{w}}(x)$ (bottom) corresponding to different moments (marked with o) of the acoustic pressure transient evolution (top), obtained for $\Delta Q / Q_{0}=10 \%$. 


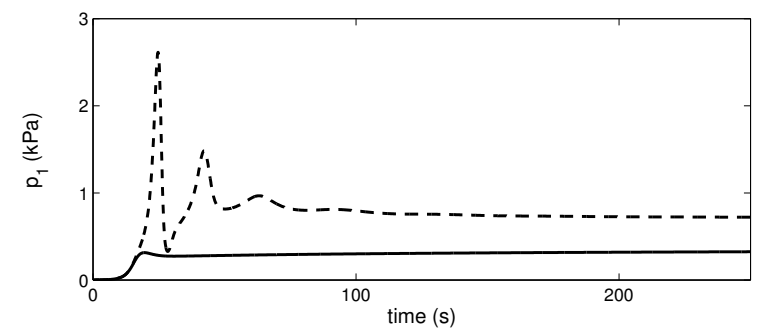

Fig. 6. Calculated evolutions with time of acoustic pressure amplitude $p_{1}$ when $\Delta Q / Q_{0}=30 \%$, whether the effect of acoustic streaming is included in the model (dashed line) or not (solid line). 